\title{
Enhancing the fungicidal activity of antibiotics: are magnetic nanoparticles the key?
}

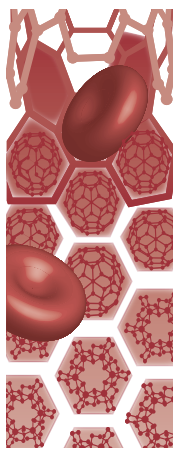

\author{
"Unique physicochemical and biological features of \\ nanomaterials make them the interesting tools for \\ developing modern antifugal methods."
}

First draft submitted: 9 February 2017; Accepted for publication: 9 March 2017; Published online: 13 July 2017

Keywords: antifungal activity • fungal infectiosn • magnetic nanoparticles $\bullet$ nanoantibiotics

Within the last 40 years, the number of fungal infections is continuously growing. More than $70 \%$ of reported cases are caused by Candida spp., especially in the population of patients with immune disorders and/or those hospitalized with serious underlying diseases [1]. Candida are the most common fungal pathogens, causing diseases ranging from superficial (oral and vaginal) to systemic such as peritonitis and meningitis candidiasis [2]. Despite a high number and diverse group of currently available antibacterial drugs, the number of active substances for treatment of pathogenic fungi is limited. Recent reports show that a growing number of Candida-mediated infections are resistant to the currently used classes of antifungal agents, particularly the azoles (where resistance has climbed most prominently) [3]. This implies the need to search for new therapeutic methods and the development of novel antifungal strategies. The increased resistance of fungi pathogens and the lack of effective treatment options for affected patients are considered to be one of the bigger challenges in current medicine. Different therapeutic approaches, including combined drug therapy, chemical structure modifications and the use of drug transporters, are employed to overcome pathogenic strain resistances [4-6]. Unique physicochemical and biological features of nanomaterials make them the interesting tools for developing modern antifugal methods $[7,8]$. Several reports indicate that nanoparticles possess the ability to inhibit the function of efflux drug transporters, promote apoptosis of fungal cells and reduce the formation of biofilm [9]. The above properties were already successfully employed during the development of novel molecules against resistant bacterial strains, where increasing activity of vancomycin-loaded nanoparticles, as compared with drug in free form against VRE-resistant enterococci, has been observed [10]. Interestingly, an analogous approach has been explored to provide new tumor-suppressing drugs against multidrug-resistant cancer cells. Indeed, the downregulation of $m d r l$ genes and the decrease of P-glycoprotein expressions were observed upon the administration of magnetic nanoparticles (MNPs) [11]. It should be emphasized that a majority of studies show that nanotechnology-based approaches might provide a method to reverse the mechanism of Candida resistance to classic antibiotics as well as expand their antimicrobial spectrum $[12,13]$. Our recently published results indicate that iron-oxide-based nanosystems provide a new strategy to overcome fungal resistance through the inactivation of catalase Cat-1. Importantly, and in agreement with other studies, we observed that a low dose of the tested nanosystems were effective to display antibiofilm activity (prevention and therapeutic efficiency), whereas

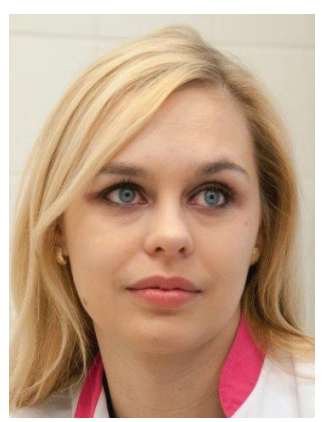

Katarzyna Niemirowicz Department of Microbiological \& Nanobiomedical Engineering, Medical University of Białystok, Białystok, Poland

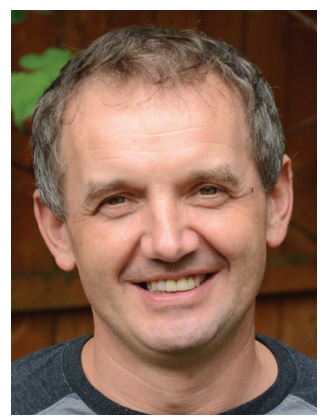

Robert Bucki

Author for correspondence: Department of Microbiological \& Nanobiomedical Engineering, Medical University of Białystok, Białystok, Poland buckirobert@gmail.com 
a five- or ten-times higher concentration of drug in free form was required to obtain similar effects [5]. It is worth emphasizing that nanotechnology-based strategies might provide a surprising outcome, as reported by Hussein-Al-Ali, who observed that a well-known antibiotic, ampicillin, when immobilized on chitosan-coated MNPs, showed activity against Candida albicans. Even if this phenomena might be partially explained by the antimicrobial properties of chitosan and MNPs (which were the components of the tested nanocomposite) [14], it should be kept in mind that a lot of the nondescript yet antifungal activities might be hidden behind the nanotechnology gate.

\section{"...more efforts should be directed to}

\section{understand the toxic aspect of nanomaterials and their pharmacological properties."}

While the immobilization of classical antibiotics on a nanoparticle's surface is not a new idea, the above works describe a new direction in this important area of nanomedicine, which is the development of new approaches for the treatment of fungal diseases. In addition, the use of MNPs as a magnetic fluid hyperthermia inductor to restrict the formation of biofilm and decrease bacteria-cell adhesion might also be used in cases of fungal-derived infections [15]. Apart from the usefulness of magnetic nanostructures in magnetic fluid hyperthermia-mediated eradication of pathogenic biofilm, there are data about the development of novel therapeutic solutions in which metallic nanoparticles are employed as enhancers of photosensitizers for antifungal photodynamic therapy [16].

An important aspect during nanoparticle creation is understanding the interactions between nanomaterials and the biological system. This will guide the

\section{References}

1 Sardi JC, Scorzoni L, Bernardi T, Fusco-Almeida AM, Mendes Giannini MJ. Candida species: current epidemiology, pathogenicity, biofilm formation, natural antifungal products and new therapeutic options. J. Med. Microbiol. 62(Pt 1), 10-24 (2013).

2 Su SY, Chao CM. Invasive Candida infections in critically ill patients. Crit. Care Med. 43(8), e322-e323 (2015).

3 Cowen LE. The evolution of fungal drug resistance: modulating the trajectory from genotype to phenotype. Nat. Rev. Microbiol. 6(3), 187-198 (2008).

4 DurnaŚ B, Wnorowska U, Pogoda K et al. Candidacidal activity of selected ceragenins and human cathelicidin LL-37 in experimental settings mimicking infection sites. PLoS ONE 11(6), e0157242 (2016).

5 Niemirowicz K, DurnaŚ B, Tokajuk G et al. Magnetic nanoparticles as a drug delivery system that enhance fungicidal activity of polyene antibiotics. Nanomedicine 2(8), 2395-2404 (2016). approaches for the safe uses of nanomaterials in the biomedical field. It is established that a nanoparticle's surface interacts with macromolecules like lipopolysaccharides, lipoteichoic acid and other proteins which are expressed on pathogen cells via hydrophobic/electrostatic/chemical adsorption [17-21]. On the other hand, this interaction also takes place with eukaryotic cells; however, recent reports have found that the difference of surface charge can help in moderating surface cytotoxic and/or antimicrobial propensities of nanoparticles. Additionally, it has recently been reported that one restriction during a nanoparticle's systemic administration is the problem associated with an affinity of blood proteins to the particle surface and the formation of a cloud (i.e. the protein corona) around it [22]. This might lead to the covering of the homing ligands that are presented on the nanoparticle's surface. Based on this observation, we suggest that the potential way to limit the 'protein cloud effect' of nanomedicine application in fungal-caused infections will be the direct administration of nanoparticles into infected area. Accordingly, more efforts should be directed to understand the toxic aspect of nanomaterials and their pharmacological properties.

\section{Financial \& competing interests disclosure}

This work was financially supported by the National Science Centre, Poland, Grants: UMO-2014/15/D/NZ6/02665 (to K Niemirowicz) and UMO-2015/17/B/NZ6/03473 (to R Bucki). The authors have no other relevant affiliations or financial involvement with any organization or entity with a financial interest in or financial conflict with the subject matter or materials discussed in the manuscript apart from those disclosed.

No writing assistance was utilized in the production of this manuscript.

6 Niemirowicz K, Piktel E, Wilczewska AZ et al. Core-shell magnetic nanoparticles display synergistic antibacterial effects against Pseudomonas aeruginosa and Staphylococcus aureus when combined with cathelicidin LL-37 or selected ceragenins. Int. J. Nanomedicine 11, 5443-5455 (2016).

7 Huh AJ, Kwon YJ. "Nanoantibiotics": a new paradigm for treating infectious diseases using nanomaterials in the antibiotics resistant era. J. Control. Release 156(2), 128-145 (2011).

8 Taylor E, Webster TJ. Reducing infections through nanotechnology and nanoparticles. Int. J. Nanomedicine 6 , 1463-1473 (2011).

9 Voltan AR, Quindós G, Alarcón KP, Fusco-Almeida AM, Mendes-Giannini MJ, Chorilli M. Fungal diseases: could nanostructured drug delivery systems be a novel paradigm for therapy? Int. J. Nanomedicine 11, 3715-3730 (2016).

10 Gu H, Ho PL, Tong E, Wang L, Xu B. Presenting Vancomycin on nanoparticles to enhance antimicrobial activities. Nano Lett. 3(9), 1261-1263 (2003). 
11 Jiang Z, Chen BA, Xia GH et al. The reversal effect of magnetic Fe3O4 nanoparticles loaded with cisplatin on SKOV3/DDP ovarian carcinoma cells. Int. J. Nanomedicine 4, 107-114 (2009).

12 Moazeni M, Kelidari HR, Saeedi M et al. Time to overcome fluconazole resistant Candida isolates: solid lipid nanoparticles as a novel antifungal drug delivery system. Colloids Surf. B Biointerfaces 142, 400-407 (2016).

13 Panwar R, Pemmaraju SC, Sharma AK, Pruthi V. Efficacy of ferulic acid encapsulated chitosan nanoparticles against Candida albicans biofilm. Microb. Pathog. 95, 21-31 (2016).

14 Hussein-Al-Ali SH, El Zowalaty ME, Hussein MZ, Geilich BM, Webster TJ. Synthesis, characterization, and antimicrobial activity of an ampicillin-conjugated magnetic nanoantibiotic for medical applications. Int. J. Nanomedicine 9, 3801-3814 (2014).

15 Park H, Park HJ, Kim JA et al. Inactivation of Pseudomonas aeruginosa PA01 biofilms by hyperthermia using superparamagnetic nanoparticles. J. Microbiol. Methods 84(1), 41-45 (2011).

16 Sherwani MA, Tufail S, Khan AA, Owais M. Gold nanoparticle-photosensitizer conjugate based photodynamic inactivation of biofilm producing cells: potential for treatment of $\mathrm{C}$. albicans infection in BALB/c mice. PLoS ONE 10(7), e0131684 (2015).

17 Niemirowicz K, Swiecicka I, Wilczewska AZ et al. Growth arrest and rapid capture of select pathogens following magnetic nanoparticle treatment. Colloids Surf. B Biointerfaces 131, 29-38 (2015).

18 Kell AJ, Stewart G, Ryan S et al. Vancomycin-modified nanoparticles for efficient targeting and preconcentration of Gram-positive and Gram-negative bacteria. ACS Nano 2(9), 1777-1788 (2008).

19 Huang YF, Wang YF, Yan XP. Amine-functionalized magnetic nanoparticles for rapid capture and removal of bacterial pathogens. Environ. Sci. Technol. 44(20), 7908-7913 (2010).

20 Shields MJ, Hahn KR, Janzen TW et al. Immunomagnetic capture of Bacillus anthracis spores from food. J. Food Prot. 75(7), 1243-1248 (2012).

21 Jin Y, Liu F, Shan C, Tong M, Hou Y. Efficient bacterial capture with amino acid modified magnetic nanoparticles. Water Res. 50, 124-134 (2014).

22 Bourzac K. News Feature: Cancer nanomedicine, reengineered. Proc. Natl Acad. Sci. USA 113(45), 12600-12603 (2016). 